\title{
Three approaches to full-spine radiograph measurement reporting
}

\author{
Takashi Takahashi $^{1}$ (I) $\cdot$ David Polly $^{2} \cdot$ Christopher T. Martin $^{2}$ \\ Received: 2 January 2019 / Accepted: 9 January 2019 / Published online: 28 January 2019 \\ (C) ISS 2019
}

\section{Background}

The result of this survey amongst members of the Society of Skeletal Radiology was no surprise for us. Based on our own experience, as a radiologist and spine surgeons, we hypothesized that sagittal parameters were uncommonly reported in radiology dictations. Although the survey does not directly answer why this under-reporting exists, the primary reason for spine surgeons to obtain full spine films is to assess sagittal balance, especially among adult spinal deformity (ASD) patients. Therefore, we thought there was a need to assess the prevalence of sagittal parameter reporting. If not reported, we wanted to highlight the importance of these parameters to the radiology community. Since these measurements are often the primary and most important reason why these studies are obtained, knowledge of these parameters' existence is crucial for radiologists who routinely dictate interpretations of these fullspine radiographs. Unless, specifically requested otherwise by ordering provider, radiologist reports should contain at least some information about sagittal spinal balance.

There are three main options for full-spine film measurement dictation for ASD. The most comprehensive option is to create a template that contains all elements of the appropriate classification system and to provide numeric measurement of each. In ASD, this is the SRS-Schwab classification, which contains four parameters including the coronal curve type, sagittal vertical axis (SVA), pelvic tilt (PT) and pelvic incidence lumbar lordosis mismatch (PI-LL). The second option is to keep all parameters, but rather than providing numeric values for each, use categorical terms. The last option is to provide a global impression of spinal balance in both coronal

Takashi Takahashi

takas005@umn.edu

1 Department of Radiology, University of Minnesota, MMC 292420 Delaware St. SE, Minneapolis, MN 55455, USA

2 Department of Orthopedic Surgery, University of Minnesota, Minneapolis, MN, USA and sagittal planes, but not to provide numeric measurements of the classification system.

\section{Full classification, numeric measurements}

This is the most comprehensive approach for measurement dictation, and requires a detailed numeric measurement of all coronal and sagittal parameters in the classification systems.

\section{Spine surgeon perspective}

The recognition and documentation of sagittal deformity is directly correlated with health-related quality of life. Appropriate identification of this deformity drives clinical treatment. Identifying the location of the deformity suggests the appropriate surgical intervention strategy. Currently, prior authorization requirements from many commercial payers specify deformity magnitude that merits surgical intervention. Some of these payers do not accept surgeon measurements but default to the radiology reports. This results in significant inefficiencies in the care process for patients who merit surgical treatment. Thus, there may be some merit to documenting in detail the relevant numeric measures. However, this also risks creating conflict with the surgeon's own measurements. Conflict with the surgeon's measurements has also been a source of insurance denials at our institution. Furthermore, the measurement of all of these parameters in heavy detail is very time consuming for the radiologist, which is financially disadvantageous. Because of these conflicts, our group has not adopted this approach.

\section{Full classification, categorical description only}

In this approach, numeric measurements are still performed by radiologists; however, the report will have categorical descriptor rather than the actual measurements. Regarding the SRSSchwab system, SVA, PT, and PI-LL have defined 
classification terms. For example, SVA less than $4 \mathrm{~cm}$ is considered insignificant, $4-9.5 \mathrm{~cm}$ positive, and over $9.5 \mathrm{~cm}$ as very positive [1]. SRS-Schwab defines coronal plane curvature over 30 degrees as abnormal and anything under as insignificant; we have created a categorical classification here of our own. We defined 10-30 degrees as "mild" because scoliosis is officially defined over 10 degrees of curvature. Over 50 degrees, we classify the curve as "severe or marked" as even after skeletal maturity, over 45 degrees often requires surgical intervention [2]. Then, between 30 and 50 we categorize as moderate.

Using the capability of a dictation system (PowerScribe, Nuance, United States), each parameter is set as a selectable field within the template, and once selected, they are converted to a classification descriptor. For instance, if SVA measures $6 \mathrm{~cm}$, one can select a category $4-9.5 \mathrm{~cm}$ under SVA section, and it had then converted to "positive" in the actual report. This feature is particularly helpful to trainees who do not routinely dictate full-spine film, as they do not need to remember each specific category. This is the version of the template that was presented at the American Society of Spine Radiology national conference in 2016.

\section{Radiologist's perspective}

We have used this template for over a year; however, as the clinical demand/volume increased, it is becoming harder to keep measuring all of these parameters. After discussing amongst MSK radiologists and spine surgeons at our institution, we have elected to use the third option.

\section{Global impression only}

The third option is the closest to a minimalist approach, yet still incorporates some information of the overall global spinal balance. Since sagittal balance assessment is a key component that surgeons assess in ASD, we have decided to keep SVA and drop PT and PI-LL. The reason is not because the other two parameters have less clinical significance, but rather that SVA was one of the easiest sagittal parameters to measure that satisfies a minimum reporting on global sagittal balance impression. This is in addition to coronal curve assessment. When providing a global impression, no detailed numeric measurements are recorded, which decreases the time investment for the radiologist, and minimizes the risk of conflicting with the surgeon's measurements. This is the version of the template we have been using for the past couple of years.

\section{Conclusions}

Sagittal parameter assessment is crucial in ASD, and knowledge of the SRS-Schwab classification is required by those who interpret full-spine radiographs. Although the best approach in regards to radiograph dictation strategy varies amongst institution and even by ordering providers, we believe it is beneficial to develop an institutional template. If necessary, different versions with varying detail could be developed, and each used according to the referring provider's request. For instance, normally we use the global impression only template to save time for routine dictation, but if the clinician requests more detailed measurements, we use the full classification, categorical description template.

With regard to AIS, these films are dictated by pediatric radiologists at our institution and they currently do not have a specific template. Since the Lenke classification is the most widely accepted system in this group, we believe that a similar approach can be employed for AIS using the Lenke classification system.

\section{Compliance with ethical standards}

Conflict of interest Takashi Takahashi: No disclosure or conflict of interest.

David Polly: No disclosure or conflict of interest.

Christopher T. Martin: No disclosure or conflict of interest.

Publisher's note Springer Nature remains neutral with regard to jurisdictional claims in published maps and institutional affiliations.

\section{References}

1. Schwab F, Ungar B, Blondel B, Buchowski J, Coe J, Deinlein D, et al. Scoliosis Research Society-Schwab adult spinal deformity classification. Spine. 2012;37(12):1077-82.

2. Sy N, Bettany-Saltikov J, Moramarco M. Evidence for conservative treatment of adolescent idiopathic scoliosis - update 2015 (mini-review). Curr Pediatr Rev. 2016;12(1):6-11. 